\title{
Análise de Velocidade para Migração em Profundidade Mediante Continuação de Velocidades em CIGs
}

\author{
G. R. Gomes (PETROBRAS \& CEP/UNICAMP), J. Schleicher (IMECC/UNICAMP \& INCT-GP), A. Novais (IMECC/UNICAMP \\ \& INCT-GP) e H. B. Santos (CEP/UNICAMP \& INCT-GP)
}

Copyright 2016, SBGf - Sociedade Brasileira de Geofísica.

Este texto foi preparado para a apresentação no Simpósio Brasileiro de Geofísica, Ouro Preto, 25 a 27 de outubro de 2016. Seu conteúdo foi revisado pelo Comitê Técnico do VII SimBGf, mas não necessariamente representa a opinião da SBGf ou de seus associados. É proibida a reprodução total ou parcial deste material para propósitos comerciais sem prévia autorização da SBGt.

\section{Resumo}

Building depth velocity models is essential for better images and reduce exploration risk. In this paper we implement the image continuation technique in the Common Image Gather (CIG) domain for application migration in depth. Through the concept of residual moveout (RMO) it was possible to develop a routine to velocity analysis. This velocity analysis allows build 2D velocity models to migrate in depth from a simple initial model. We demonstrate the method's ability to construct acceptable models in depth without the need for prior information. The results were initially obtained for a synthetic data corresponding to a model consisting of three layers and was then applied to the data Marmousoft (Billette et al., 2003).

\section{Introdução}

Muitos autores têm proposto Migration Velocity Analysis, MVA, como forma de obter o modelo de velocidades para migração. Os métodos discutidos abrangem uma gama que começa numa simples comparação de imagens de afastamento comum (Gardner, 1974), passa por extrapolação de famílias CMP (Yilmaz and Chambers, 1984), migrações tentativas seguido de empilhamento (Fowler, 1985) e análise da profundidade do focamento (Jeannot et al., 1986) para terminar em análise do moveout residual (residual moveout, RMO) dentro da família de ponto comum (Common Image Gather, CIG) no domínio do ângulo comum (angle-domain $\mathrm{CIG}$ ), $\mathrm{ADCIG}$, ver Biondi and Symes (2004).

Por causa de sua clareza conceitual e simplicidade, a análise RMO se tornou a ferramenta favorita para MVA. Recentemente foi proposto um novo método de MVA para migração em tempo que faz uso da correção do RMO por variação da velocidade de migração. Este método de MVA propõe-se a começar a análise com um modelo inicial simples, sem a necessidade de especificar nele determinadas características do modelo procurado. Sendo assim, este método se distingue fundamentalmente dos métodos tomográficos ou de inversão full waveform, que necessitam de um bom modelo inicial para garantir a sua convergência.

Schleicher et al. (2008) construíram uma ferramenta para análise RMO usando uma implementação de diferenças finitas de uma equação diferencial parcial (EDP). A idéia é propagar os eventos dentro dos CIGs até que fiquem horizontais, usando a EDP deduzida especificamente para este fim. Esta EDP que descreve o deslocamento dos eventos em função da velocidade de migração foi denominada de equação da onda imagem (Goldin (1994), Fomel (1994, 1997) e Hubral et al. (1996)). Assim, podese encontrar, para cada evento identificável, um valor da velocidade que horizontaliza-o. Após migração com o modelo assim construído, o procedimento pode ser repetido para melhorá-lo.

O maior atrativo da MVA por propagação da imagem dentro do CIG, é sua capacidade de construir uma imagem a partir de um modelo inicial muito básico. No trabalho original de Schleicher et al. (2008), um modelo constante com a velocidade de água foi suficiente para recuperar um modelo de velocidade aceitável para migração em tempo dos dados Marmousoft (Billette et al., 2003).

A versão do método implementada por Schleicher et al. (2008) é restrita à migração em tempo. Este método de migração somente é aplicável em meios com pouca variação lateral. Sendo assim, a imagem migrada do modelo Marmousoft na região central, onde há falhas e altas veriaçãoes laterais da velocidade, fica prejudicada.

Por outro lado, Schleicher and Biloti (2007) também deduziram uma versão da equação da onda imagem para a migração em profundidade. Neste trabalho, vamos mostrar resultados obtidos com o método de diferenças finitas para esta EDP em profundidade, para depois aplicar o método de MVA neste domínio. Será mostrado um processo construção do modelo 2D para um processo adequado em profundidade (Schleicher et al., 2004). Os resultados foram obtidos inicialmente para um dado sintético, um modelo de três camadas. Em seguida foi aplicado ao dado do Marmousoft (Billette et al., 2003). Os resultados são animadores, visto que na primeira iteração é obtido um modelo razoável para migração em profundidade.

\section{Metodologia}

A construção do modelo de velocidade é feita através da continuação da imagem em profundidade no domínio do CIG. A EDP que descreve o deslocamento em profundidade dos eventos no CIG foi obtida por (Schleicher and Biloti, 2007). Partindo da expressão de que descreve a pseudo-profundidade migrada de um refletor horizontal em um meio homogêneo (Schleicher and Biloti, 2007) chegaram a expressão:

$$
\frac{\partial P}{\partial z}+\frac{v z}{h^{2}+z^{2}} \frac{\partial P}{\partial v}=0
$$


onde $P$ é a amplitude do evento em profundidade, $v$ é a velocidade de migração, $h$ é o meio-afastamento e $z$ é a pseudo-profundidade migrada. A equação (1) tem a forma matemática de uma equação de onda one-way 1D onde $v$ faz o papel da variável temporal e $\left(h^{2}+z^{2}\right) / v z$ faz o papel da velocidade de propagação. A equação (1) é discretizada para solução por diferenças finitas conforme análise feita em Schleicher et al. (2008).

A partir do esquema de diferenças finitas é possível continuar um CIG da velocidade de partida para uma outra que se deseja. Na continuação é definida a velocidade de partida (referência) igual a velocidade constante que gerou o CIG. Assim, partindo de um CIG obtido com a migração com velocidade constante pode-se determinar - CIG em outra velocidade sem precisar migrar. A variação da imagem no CIG por conta da variação na velocidade reflete no moveout da curva do evento. Se a velocidade de continuação for igual a velocidade correta do evento a curva fica horizontal, se não, a curva apresenta moveout. Este tipo de análise é conhecido como análise RMO (acrônimo de Residual MoveOut). A continuação da imagem no CIG permite variar o moveout dos eventos no CIG mediante variação de velocidade. Com isto, o que se pretende é fazer com que os eventos fiquem horizontais e daí extrair informação de profundidade e velocidade do evento. Esta é a base para análise de velocidade aqui desenvolvida.

A velocidade média de migração em profundidade é descrita pelo inverso da média sobre a vagarozidade (Schleicher et al., 2004). Deste fato é possível converter as velocidade médias em intervalares. Supondo que as velocidades médias obtidas para dois eventos em $z_{j}$ e $z_{j+1}$, abaixo da profundidade $z_{0}$, são respectivamente $V_{m}^{j}$ e $V_{m}^{j+1}$ então a expressão para velocidade intervalar é:

$$
\frac{1}{V_{r}^{j}}=\frac{\left(\frac{z_{j}-z_{0}}{V_{m}^{j}}-\frac{z_{j-1}-z_{0}}{V_{m}^{j-1}}\right)}{\left(z_{j}-z_{j-1}\right)} .
$$

A velocidade intervalar obtida assim contém um erro associado a determinação da velocidade média, pois a equação 1 se baseia no modelo de camada horizontal. Este erro pode ser verificado através do moveout dos ClGs. Se os eventos não estiverem devidamente horizontais é necessário melhorar o modelo. Uma alternativa para melhorar o modelo é usar o dado migrado para uma nova continuação. Neste caso a velocidade de partida não é mais constante, mas impõe-se que seja, fornecendo um valor constante de velocidade para fazer a continuação. Sendo assim, as novas velocidades médias são relativisadas e giram em torno do valor constante escolhido para continuação. A diferença entre as novas velocidades médias e o valor escolhido para continuação pode ser utilizado para atualizar o modelo. Então supondo que $V_{m, i}^{j}$ e $V_{m, i+1}^{j}$ sejam dois valores de velocidade média em duas iterações subsequentes $i$ e $i+1$, para o evento $j$ temos as seguintes expressões para atualização:

$$
\begin{aligned}
& \frac{1}{V_{n}} \approx \frac{1}{V_{m, i+1}^{j}}=\frac{1}{V_{m, i}^{j}+V_{f}^{j}-V_{c}} \mathrm{e} \\
& \frac{1}{V_{n}} \approx \frac{1}{V_{m, i+1}^{j}}=\frac{1}{V_{m, i}^{j}}+\frac{1}{V_{f}^{j}}-\frac{1}{V_{c}},
\end{aligned}
$$

onde $V_{f}^{j}$ é a velocidade média relativa no j-ésimo evento, $V_{c}$ é o valor de velocidade constante utilizado na continuação e $V_{n}$ é velocidade de migração ótima (que horizontaliza o evento perfeitamente). Na expressão (3) estamos atualizando as velocidades equanto que na expressão (4) estamos atualizando as vagarosidades.

O processo iterativo para construção e atualização do modelo é similar ao descrito em (Schleicher et al., 2008). O fluxo de construção do modelo é composto dos seguintes passos:

1. Migre os dados usando uma velocidade constante qualquer e ordene os dados em CIGs.

2. Aplique a continuação de determinados CIGs.

3. Determine as velocidades e profundidades de horizontalização.

4. Construa um novo modelo de migração utilizando (2). Se for a primeira execução, é feita apenas o preenchimento do modelo. Se for uma execução subsequente, é necessário fazer a atualização utilizando uma das expressões (3) ou (4).

5. Migre os dados usando este novo modelo.

6. Se os CIGs não estiverem satisfatoriamente horizontalizados aplique a continuação mais uma vez.

7. Repita os passos 3. a 6. até que a horizontalização dos eventos nos CIGs esteja satisfatória.

\section{Resultados}

Geramos um dado sintético utilizando o traçamento de raio paraxial do programa SM88Modeling implementado na interface GêBR (Biloti, 2010) no modelo da Figura 1. O dado é regular e consta de 50 seções de offset constante, com intervalo entre as seções de $100 \mathrm{~m}$, o mais próximo com $50 \mathrm{~m}$ e o mais longo com $4950 \mathrm{~m}$, e intervalo entre os CMPs de $50 \mathrm{~m}$. Os dados foram migrados

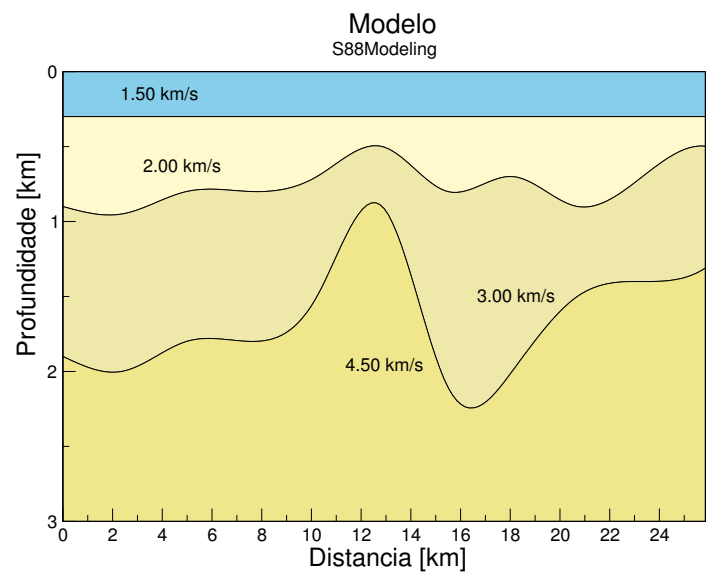

Figura 1: Modelo de velocidade utilizado para gerar dados sintéticos com interfaces suaves para traçamento de raio.

com velocidade constante de $2000 \mathrm{~m} / \mathrm{s}$, de acordo com o 
primeiro passo do fluxo de construção. De acordo com o passo 2, selecionamos $51 \mathrm{ClGs}$ a cada $500 \mathrm{~m}$ para serem continuados na faixa de velocidades de $1400 \mathrm{~m} / \mathrm{s}$ a $4000 \mathrm{~m} / \mathrm{s}$, com velocidade de partida de $2000 \mathrm{~m} / \mathrm{s}$. O semblance do CIG 7525 é mostrado na Figura 2. Na seção

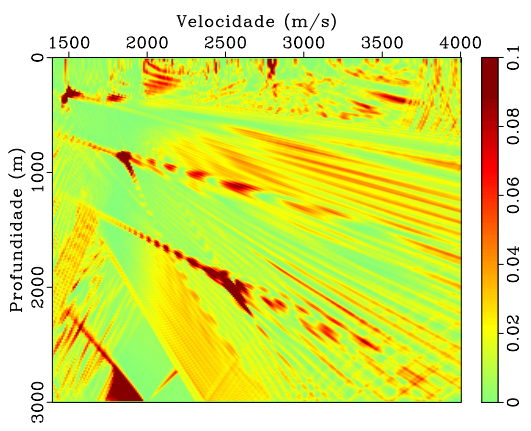

Figura 2: Seção de velocidade obtida através do semblance convencional dos CIGs continuados.

da Figura 2 bem como nas outras 50 seções de semblance foi possível definir, com pick manual, os melhores valores de profundiade e velocidade média e construir o modelo. A Tabela 1 mostra os valores obtidos de pick referente ao CIG 7525 e a Tabela 2 mostra os valores do modelo obtido de acordo com (2) com os respectivos erros relativo.

\begin{tabular}{||c|c||}
\hline Profundidade $(\mathrm{m})$ & Velocidade Média $(\mathrm{m} / \mathrm{s})$ \\
\hline 323.48 & 1498.31 \\
\hline 902.61 & 1884.75 \\
\hline 1961.74 & 2542.37 \\
\hline
\end{tabular}

Tabela 1: Valores de profundidade e velocidade média obtidos com pick da Figura 1.

\begin{tabular}{||c|c|c|c||}
\hline Profundidade $(\mathrm{m})$ & Erro & Velocidade $(\mathrm{m} / \mathrm{s})$ & Erro \\
\hline 323.48 & $8 \%$ & 1498.31 & $-0.1 \%$ \\
\hline 902.61 & $13 \%$ & 2201.97 & $10 \%$ \\
\hline 1961.74 & $9 \%$ & 3618.27 & $20 \%$ \\
\hline
\end{tabular}

Tabela 2: Modelo construído para o CIG 7525 obtidos a partir dos picks na Figura 1. São mostrados os valores da profundidade do refletor e a Velocidade Intervalar com seus respectivos erros relativos.

Como os picks são feitos somente em determinados CIGs foi necessário fazer uma interpolação linear entre as posições para preenchimento do modelo. O resultado do primeiro modelo é mostrado na Figura $3(\mathrm{~b})$. O dado foi migrado com este modelo de acordo com o passo 5 e os CIGs migração são mostrados na Figura 4. Este modelo tem a mesma morfologia do modelo real e os valores de velocidade próximos. Isto demonstra o quanto o método é promissor. Já que alguns valores de velocidade e profundidade têm o erro relativo além dos $10 \%$ há margem para melhoria. $O$ erro no modelo também pode ser constatado através dos CIGs da Figura 4 que mostram que os eventos estão voltados para baixo, ou seja os valores de velocidade ainda estão altos.
Os CIGs obtidos com esta migração são submetidos a nova continuação com velocidade de referência de $2000 \mathrm{~m} / \mathrm{s}$ e feita nova análise de velocidade para atualização do modelo. A Figura 3(c) mostra o modelo da segunda iteração e a Figura 3(d) da terceira iteração. Uma análise pontual mostra que existem valores na terceira iteração que se distanciaram do valor real. Isto é perfeitamente possível em regiões com variação lateral forte de velocidade e por conta da aproximação que estamos fazendo. No entanto, por inspeção vizual no mapa de cores do modelo nota-se que o modelo da terceira iteração está mais próximo do real que os outros dois. Assim sendo, reconhecemos que o resultado está convergindo. Isso pode ser constatado com a análise dos ClGs obtidos de cada modelo (ver Figuras 4, 5 e 6). Para o sucesso e robustez da construção do modelo é importante ter em mente a estratégia de layer-stripping que é corrigir o máximo possível os eventos mais rasos antes de tentar corrigir os mais profundos. Neste caso, para não atualizar os valores de profundidade e velocidade calculados para eventos já corrigidos basta fazer o pick na profundidade do evento e na velocidade igual a de referência. Com isto, não é gerado um valor de atualização.

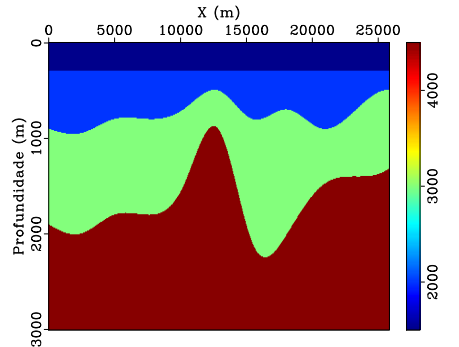

(a)

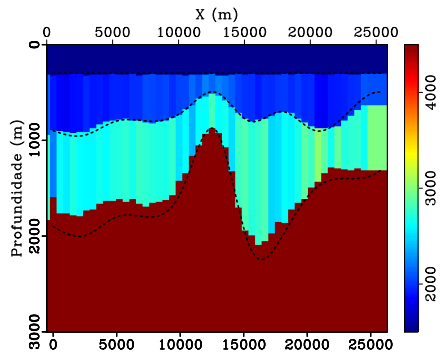

(c)

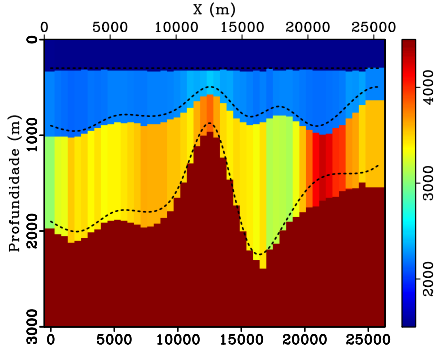

(b)

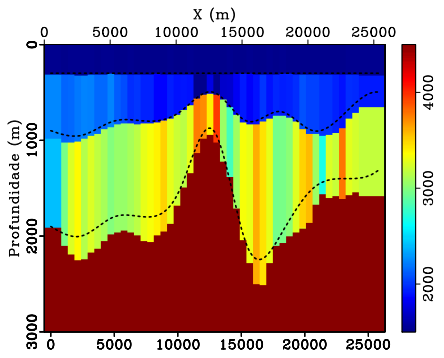

(d)
Figura 3: Evolução dos modelos de velocidade: (a) modelo real, (b) primeiro modelo, (c) segundo modelo e (d) terceiro modelo. Neste caso foi feita atualização das velocidades de acordo com a expressão (3)

Utilizamos também os dados do Marmousoft para testar o método. O dado consta de 96 seções de offset constante, com intervalo entre as seções de $25 \mathrm{~m}$, o mais próximo tem $100 \mathrm{~m}$ e o mais longo $2475 \mathrm{~m}$, e intervalo entre os CMPs de $12.5 \mathrm{~m}$. Selecionamos $35 \mathrm{CIGs}$ a cada $250 \mathrm{~m}$ para serem continuados na faixa de velocidades de $1400 \mathrm{~m} / \mathrm{s}$ a $4000 \mathrm{~m} / \mathrm{s}$, com velocidade de referência de $2000 \mathrm{~m} / \mathrm{s}$. As seções de semblance de alguns CIGs podem ser vistas na Figura 7. As seções de semblance nos CIGs 5500 e 6500 , que situam-se na parte central do modelo e abaixo de $1000 \mathrm{~m}$ de profundidade, não têm os picos bem definidos como nos outros por conta 


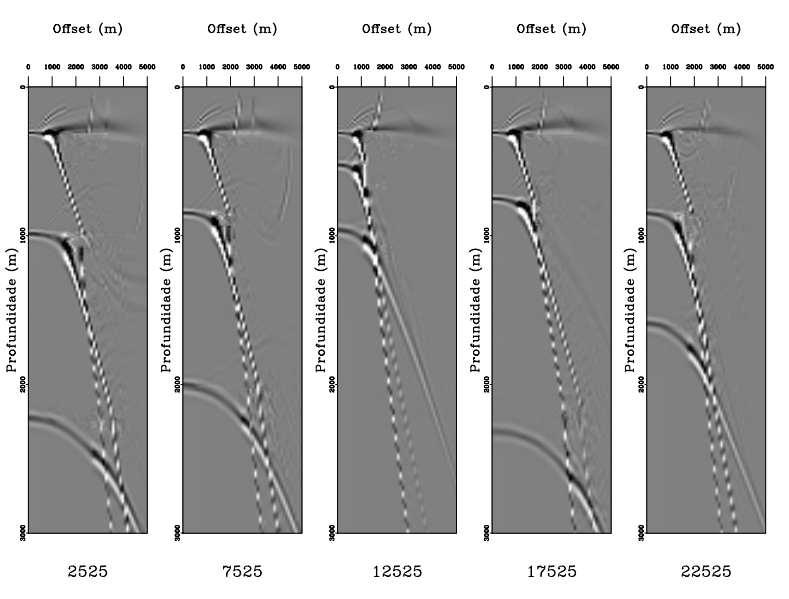

Figura 4: CIGs para primeira iteração. Obtido a partir da migração com modelo Figura 3(b).

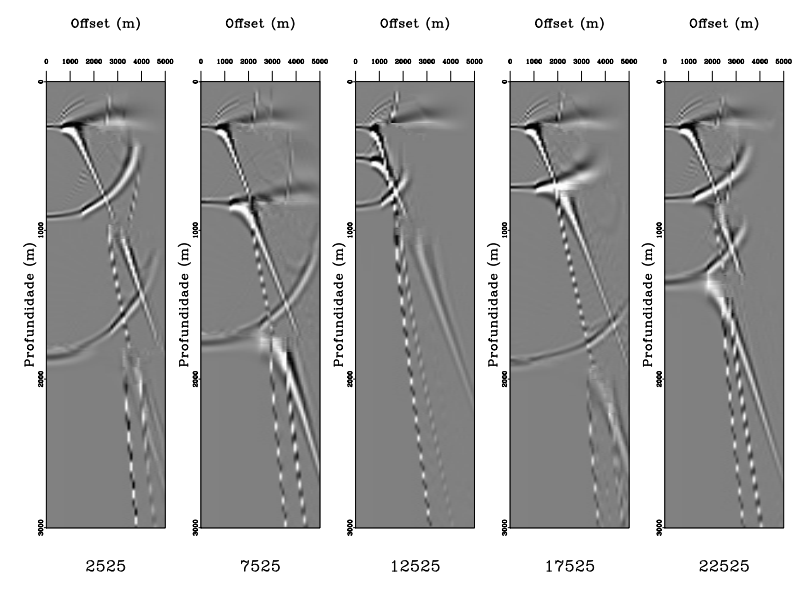

Figura 5: CIGs para segunda iteração. Obtido a partir da migração com modelo da Figura 3(c).

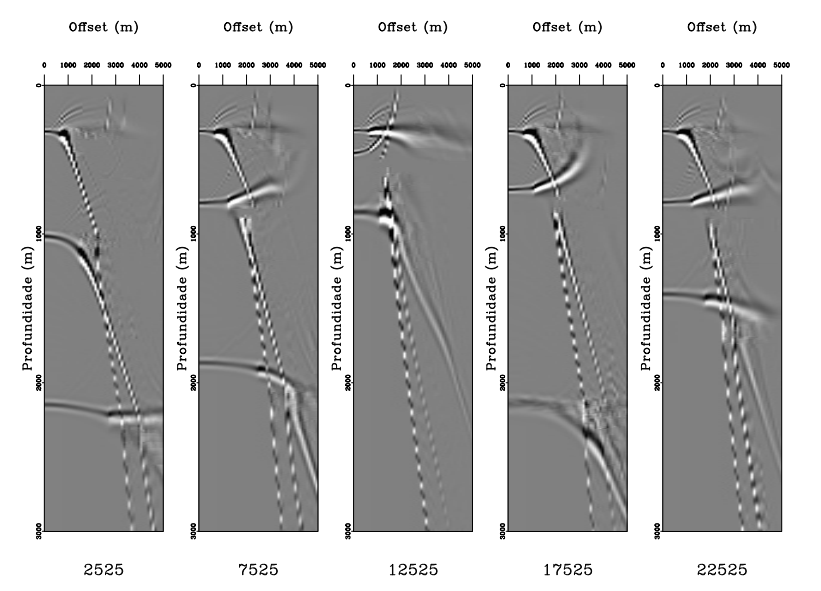

Figura 6: CIGs para terceira iteração. Obtido a partir da migração com modelo da Figura 3(d). da complexidade do meio. Esta ambiguidade aumenta as chances de erro na geração do modelo nesta região. O modelo obtido a partir dessa primeira análise de velocidade é mostrado na Figura 8 . Os CIGs obtidos a partir da migração deste modelo é mostrado na Figura 8. A imagem obtida a partir do empilhamento dos CIGs corrigidos é mostrada na Figura 10. O resultado obtido mostra que até $2000 \mathrm{~m}$ os refletores estão bem definidos com as estruturas corretamente posicionadas. A imagem das falhas é reforçada. Além de $2000 \mathrm{~m}$, e na parte central pode-se notar a imagem distorcida como um pullup dos eventos induzido pela ambiguidade no semblance já dito antes, mas também pela propagação de erro no modelo acima (supondo que exista). De qualquer forma, o resultado é bom para um primeiro modelo o que demonstra o quanto é promissor o método.

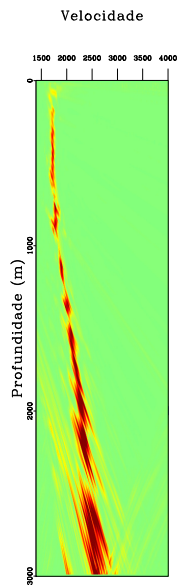

3500

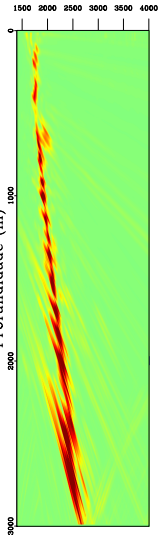

4500

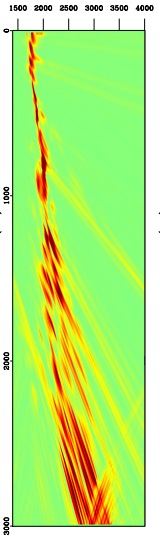

5500

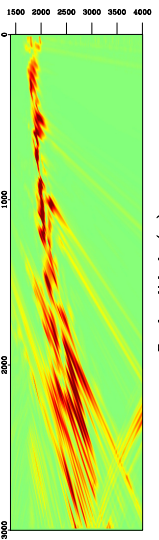

6500
Velocidade

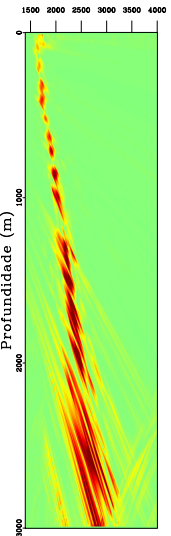

7500
Figura 7: Semblances obtidos para o dado Marmousoft.

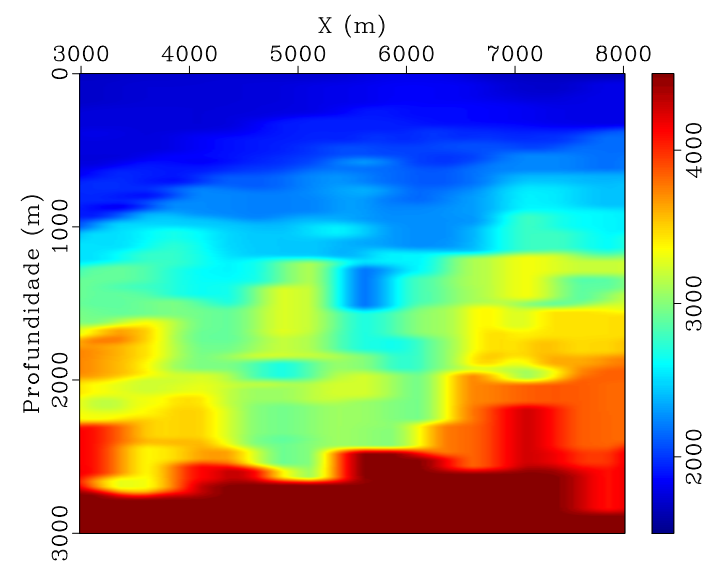

Figura 8: Modelo de velocidade para o dado Marmousoft: primeiro modelo.

\section{Conclusões}

O trabalho mostra que foi implementado uma técnica para construção do modelo de velocidade em profundidade utilizando continuação da imagem no CIG. Além disso, é apresentada a opção de atualizar esse primeiro modelo a 


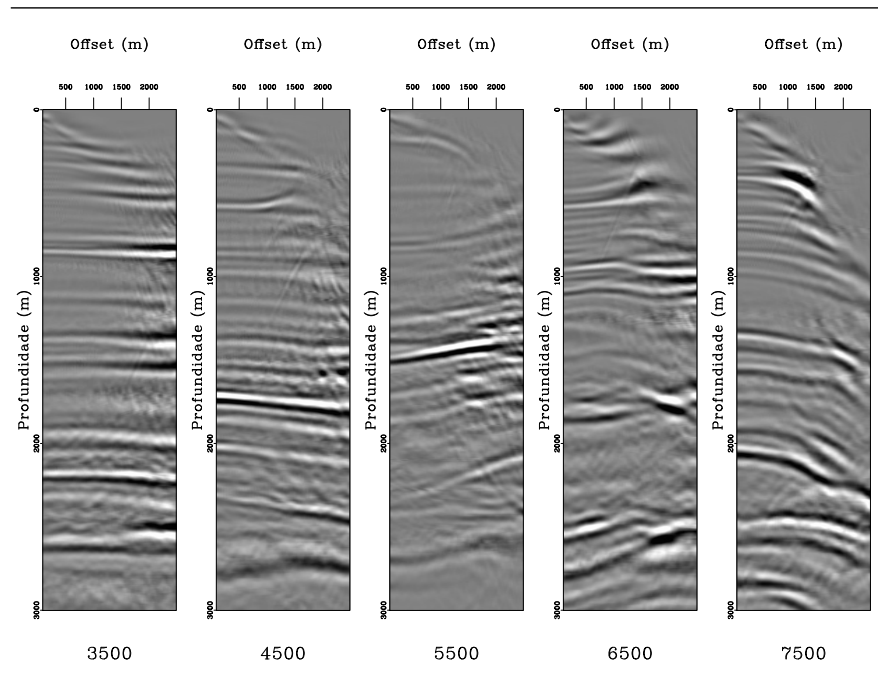

Figura 9: CIGs do dado Marmousoft para primeira iteração. Obtido a partir da migração com modelo Figura 8.

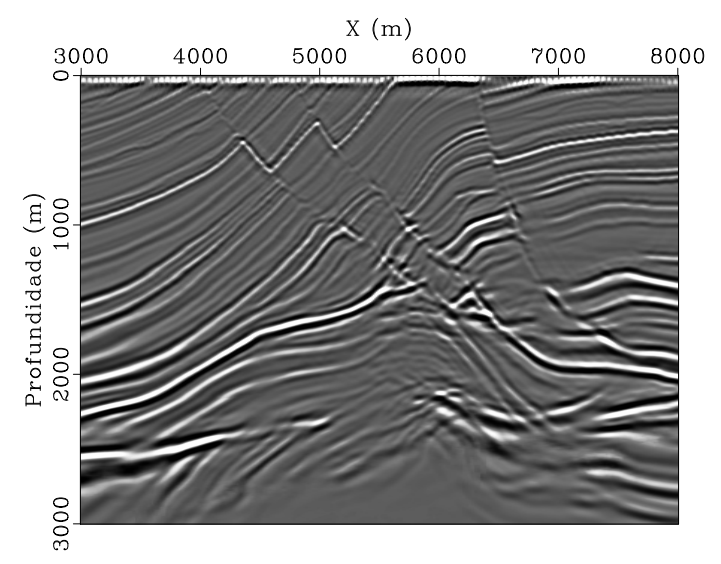

Figura 10: Resultado da migração (empilhado) para o dado Marmousoft para o modelo Figura 8.

fim de melhorá-lo através de um processo iterativo. Essa técnica apresenta resultados promissores tendo em vista que uma primeira iteração, partindo de um modelo de velocidade constante, já é possível obter a morfologia do modelo que está sendo invertido. Também foi mostrado no exemplo sintético que o processo iterativo converge para um modelo melhor e isso é constatado através dos CIGs. A imagem obtida (seção empilhada) a partir do primeiro modelo, para o caso do Marmousoft, apresenta de um modo geral a estruturação correta dos eventos e o destaque das falhas. Por fim, a técnica pode ser empregada para reduzir o risco no processo exploratório através da geração expedita de imagens de qualidade.

\section{Acknowledgements}

Essa pesquisa foi apoiada pelo CNPQ, pela Petrobras e também pelo patrocínio do consórcio WIT.

\section{Referências}

Billette, F., S. L. Bégat, P. Podvin, and G. Lambaré, 2003, Pratical aspects and applications of $2 d$ stereotomography: Geophysics, 68, 1008-1021.

Biloti, R., 2010, S88 modeling. Department of Applied Mathematics, IMECC/UNICAMP. (S88 Modeling employs Seis88 avaiable in http://sw3d.mff.cuni.cz/ package developed by Vlatislav Cerveny and Ivan Psencik. version 3. It is part of ToSCo Project).

Biondi, B., and W. W. Symes, 2004, Angle-domain common-image gathers for migration velocity analysis by wavefield-continuation imaging: Geophysics, 69, 12831298.

Fomel, S., 1994, Method of velocity continuation in the problem of seismic time migration: Russian Geology and Geophysics, 35, 100-111.

-1997 , Velocity continuation and the anatomy of residual prestack migration: Expanded Abstract, 67th Annual International Meeting, SEG, 1762-1765.

Fowler, P., 1985, Migration velocity analysis by optimization:linear theory: SEP Report, 44, 1-20.

Goldin, S. V., 1994, Superposition and continuation of transformations used in seismic migration: Russian Geology and Geophysics, 35, 109-121.

Hubral, P., M. Tygel, and J. Schleicher, 1996, Seismic image waves: Geophysical Journal International, 431442.

Jeannot, J. P., J. P. Faye, and E. Denelle, 1986, Prestack migration velocities from focusing depth analysis: Expanded Abstract, 56th Annual International Meeting of SEG, SEG, 438-440.

Schleicher, J., and R. Biloti, 2007, Dip correction for coherence-based time migration velocity analysis: Geophysics, 72, S41-S48.

Schleicher, J., J. C. Costa, and A. Novais, 2008, Timemigration velocity analysis by image-wave propagation of common-image gathers: Geophysics, 73, VE161VE171.

Schleicher, J., A. Novais, and F. P. Munerato, 2004, Migration velocity analysis by depth image-wave remigration: first results: Geophysical Prospecting, 52, 559-573.

Yilmaz, O., and R. Chambers, 1984, Migration velocity analysis by wave-field extrapolation: Geophysics, 49, 1664-1674. 